\title{
Left Atrial Appendage Circular RNAs Are New Predictors of Atrial Fibrillation Recurrence After Surgical Ablation in Valvular Atrial Fibrillation Patients
}

\author{
Xiyu Zhu, MD, ${ }^{1}$ Yali Wang, MD,${ }^{1}$ Ran Mo, MD,${ }^{2}$ Hoshun Chong, MD,${ }^{1}$ Hailong Cao, MD,${ }^{1}$ Fudong Fan, \\ MD, ${ }^{1}$ Qing Zhou, MD, ${ }^{1}$ Dongjin Wang, $\mathrm{MD}^{1}$ \\ ${ }^{1}$ Department of Cardio-Thoracic Surgery, Nanjing Drum Tower Hospital, The Affiliated Hospital of Nanjing University Medical \\ School, Nanjing, Jiangsu Province, China; ${ }^{2}$ Department of Burn \& Plastic Surgery, Nanjing Drum Tower Hospital, The Affiliated \\ Hospital of Nanjing University Medical School, Nanjing, Jiangsu Province, China
}

\section{ABSTRACT}

Background: Atrial fibrillation (AF) recurrence after ablation will increase mortality and morbidity during follow up. We attempted to evaluate the relationship between circular RNAs (circRNA) and AF recurrence to establish a predictive model for early intervention.

Methods: Patients who received surgical ablation retrospectively were analyzed. The expression of circRNAs were detected in the left atrial appendage. The independent risk factors of late recurrence were analyzed by multivariate analysis. The predictive model was visualized by Nomogram and tested by receiver operating characteristic curve and calibration plot. Kaplan-Meier plot was used to compare the rate of freedom from AF recurrence after surgery. The relationships between circRNAs and clinical characteristics were detected by Spearman's correlation analysis.

Results: A total of 136 patients were enrolled from September 2018 to June 2019, 55 patients experienced late recurrence during one-year follow up. Increased age, longer $\mathrm{AF}$ duration and increased circ 81906-RYR2, circ 44782LAMA2, circ 418-KCNN2 and circ 35880-ANO5 were detected in recurrent patients. Multivariate analysis revealed that increased age (odds ratio $(\mathrm{OR})=1.072, P=0.006)$, longer AF duration $(\mathrm{OR}=1.007, P=0.036)$ and increased circ $81906-$ RYR2 (OR=2.210, $P<0.001)$ were independent risk factors for late recurrence. Area under the curve was 0.77, and the cut-off value was 70 points of the predictive model. KaplanMeier plots showed that patients over 70 points tended to experience $\mathrm{AF}$ recurrence.

Conclusion: Circ 81906-RYR2 could be a new predictor of late recurrence after surgical ablation. A predictive model consists of age, atrial fibrillation duration, and circ 81906RYR2 was alternative for early intervention of AF recurrence.

Received Fuly 13, 2021; accepted September 3, 2021.

Correspondence: Dr: Dongjin Wang, No.321 Zhongshan Rd, Nanjing, Fiangsu Province, China, Telephone +86 (025) 83106666-60710 (e-mail: wangdongjin@njglyy.com).

\section{INTRODUCTION}

Surgical radiofrequency ablation accompanied with valve surgery is an effective method to restore sinus rhythm (SR) in valvular atrial fibrillation (AF) patients [Baumgartner 2017]. Restoration of sinus rhythm will help to decrease the incidence of heart failure, re-hospitalization and thrombotic events during follow up [Rattka 2020]. However, the restoration rate after surgery varied among different centers $(65 \%$ 95\%) [Fayad 2005; Geidel 2006; Gammie 2008; Gillinov 2006]. A real-world nationwide study also showed the rate of $\mathrm{AF}$ recurrence range from 31\% to $45 \%$ [Pallisgaard 2018]. The outcome of patients with persistent or permanent $\mathrm{AF}$ was worse than patients with paroxysmal $\mathrm{AF}$ after ablation therapy [Balk 2010]. Several risk factors have been identified in the former studies, such as advanced age, concomitant cardiovascular disease, and the length of AF duration [Themistoclakis 2008; Chen 2018; Khan 2020] to predict the risk of postoperative $\mathrm{AF}$ recurrence. It is helpful to find some new biomarkers to predict $\mathrm{AF}$ recurrence after surgical ablation in $\mathrm{AF}$ patients with valvular heart disease (VHD) and start early therapeutic intervention for those suffering from $\mathrm{AF}$ recurrence.

Circular RNA (CircRNA) is a kind of non-coding RNA that has attracted much research focus in the past decade [Nigro 1991]. Research has revealed that circRNAs can be used as clinical predictors for central nervous disease [Westholm 2014] and cancers [Han 2018]. Our former study found several differentially expressed circRNAs in AF patients with VHD [Zhu 2020], however, whether these circRNAs can be used as clinical biomarkers for the outcome of surgical ablation still needs further evaluation. The aim of this study was to evaluate the prognostic value of circRNAs for the outcome of surgical ablation in persistent $\mathrm{AF}$ patients with VHD.

\section{MATERIALS AND METHODS}

Ethics statement: This study was approved by the ethics committee of the Nanjing Drum Tower Hospital (approval number: 2016-151-01). Written informed consent was obtained from all patients enrolled in this study.

Patient enrollment: A total of 150 valvular AF patients were admitted to our center from September 2018 to June 
2019. Exclusion criteria were as follows: 1) age of patients younger than 18 or more than 75 years old, 2) patients with aortic diseases, infective endocarditis, congenital heart diseases or acute myocardial infraction, 3) non-valvular AF, 4) patients died during perioperative period. Preoperative transthoracic echocardiography (TTE) was performed to evaluate the subtype of valve diseases, degree of valve diseases, left atrial diameter (LAD), and left ventricular ejection fraction (LVEF). Persistent AF was diagnosed by the preoperative 24-hour Holter examination in patients with continuous AF over three months. Patients over 50 years needed to finish coronary angiography to evaluate the coronary artery lesion. Demographic data (including age, gender, and body mass index), cardiovascular risk factors (hypertension, diabetes mellitus, chronic kidney disease, smoke, or alcohol abuse), concomitant diseases, results of TTE and intro-operative data (operation time, cardiopulmonary bypass time, aortic crossclamp time, valve repair or replacement, and type of artificial valve) were recorded for further exploration. Remote ECG monitoring was used for all patients in the first five days after operation. Beta blocker or amiodarone was used to maintain the heart rate between 60 and $70 \mathrm{bpm}$. All patients were discharged without complications and received more than one year follow up. Telephone interviews were performed monthly, and all patients were required to finish the 24-hour Holter examination during their visit to the outpatient clinic at three months, six months, and one year after operation. Patients were divided into two groups as sinus rhythm maintenance (SRM) and atrial fibrillation recurrence (AFR), according to the results of the 24 hours Holter examination. Patients who experienced AF events in three months received at least once cardioversion and following antiarrhythmic drug therapy.

Surgical procedures of radiofrequency ablation: Surgical radiofrequency ablation was performed in all enrolled patients. Bi-atrial ablations were achieved by using the cardioblate surgical ablation system (Medtronic, Minneapolis, USA) and following the Cox-Maze IV procedure [Robertson

Table 1. Clinical characteristics of valvular PeAF patients in 3 months follow up

\begin{tabular}{|c|c|c|c|}
\hline Age (year) & $58.9 \pm 9.6$ & $61.4 \pm 8.0$ & 0.098 \\
\hline Body mass index $\left(\mathrm{kg} / \mathrm{m}^{2}\right)$ & $23.3 \pm 3.6$ & $24.4 \pm 3.3$ & 0.055 \\
\hline AF duration (month) & $41.0 \pm 60.6$ & $61.2 \pm 77.6$ & 0.023 \\
\hline Hypertension (n, \%) & $22(30.6 \%)$ & $25(39.1 \%)$ & 0.367 \\
\hline Diabetes mellitus (n, \%) & $3(4.2 \%)$ & $5(7.8 \%)$ & 0.475 \\
\hline Chronic kidney disease (n, \%) & $1(1.4 \%)$ & $2(3.1 \%)$ & 0.601 \\
\hline Smoke abuse (n, \%) & $7(9.7 \%)$ & $5(7.8 \%)$ & 0.769 \\
\hline Alcohol abuse (n, \%) & $5(6.9 \%)$ & $2(3.1 \%)$ & 0.447 \\
\hline Left atria diameter $(\mathrm{cm})$ & $5.1 \pm 0.9$ & $5.5 \pm 1.1$ & 0.024 \\
\hline Ejection fraction $(\mathrm{n}, \%)$ & $52.4 \pm 6.9$ & $53.0 \pm 7.8$ & 0.403 \\
\hline Rheumatic valvular disease (n, \%) & $40(55.6 \%)$ & $32(50.0 \%)$ & 0.606 \\
\hline \multicolumn{4}{|l|}{ NYHA $†$ Class } \\
\hline Class I (n) & 1 & 4 & \\
\hline Class II (n) & 34 & 24 & 0.343 \\
\hline Class III (n) & 36 & 34 & \\
\hline Class IV (n) & 1 & 2 & \\
\hline $\mathrm{BNP} \ddagger(\mathrm{pg} / \mathrm{mL})$ & $345.5 \pm 301.7$ & $319.2 \pm 301.3$ & 0.769 \\
\hline
\end{tabular}

‡BNP, brain natriuretic peptide; §CRP, C-reactive protein; ๆeGFR, estimated glomerular filtration rate; †NYHA, New York heart association 
2014]. Left atrial appendage (LAA) was resected during the operation to avoid thrombotic events. Valve replacement or repair were performed in all enrolled patients. Coronary artery bypass graft was operated in patients with coronary artery disease. In summary, circumferential pulmonary vein isolation and the right atrial ablation were performed before aortic cross-clamping, then the left atrial ablation was performed, according to the Cox-Maze IV procedure. The ablation energy was set between 25 and 30 Watts, according to the thickness of the atrium. There were 40 mitral valve repair, 10 aortic valve repair, 125 tricuspid valve repair, 66 mitral valve replacement, 26 aortic valve replacement, one tricuspid valve replacement, and 13 coronary artery bypass grafts performed over the same period during surgical ablation.

Expression of circRNAs in LAA tissue: LAA was resected during surgery and divided into two parts, of which one immediately was frozen into liquid nitrogen for RNA isolation and the other was prepared for pathological analysis.

The expression of circRNAs in LAA were detected by using quantitative real-time reverse transcription polymerase chain reaction (qRT-PCR). The total RNA was extracted from LAA sample using TRIzol Reagent (Cat. No 15596018, ThermoFisher Scientific), according to the manufacturer's protocol. The quantity and quality of total RNA were detected by NanoDrop One (ThermoFisher Scientific) and agarose gel electrophoresis, then it was reverse transcribed to synthesize cDNA by using HiScript II Q Select RT SuperMix (Cat. No R232-01, Vazyme). The expression of circRNAs (circ 81906-RYR2, circ 44782-LAMA2, circ 418-KCNN2, circ 35880-ANO5, and circ 255-ITGA7) was measured by qRT-PCR with ChamQ Universal SYBR qPCR Master Mix (Cat. No Q711-02, Vazyme) and LightCycler 480 Instrument II (Roche Molecular System, Inc). GAPDH was selected as the internal reference, and the relative expression of circRNA was calculated by the $2^{-\triangle \Delta C T}$ method. The primers of circRNAs were listed in the Supplementary Table 1. (Supplementary Table 1)

Statistical analysis: Numerical variables were described as mean \pm standard deviation (SD), categorical variables were presented by count. Statistical analysis was performed by using SPSS version 23.0. Continuous variables were compared by Student's t test or Mann-Whitney U test, according to the normality test. Chi-square test was used to analyze the categorical variables, and fisher's exact was used when sample size was less than five. Multivariate logistic regression was performed to find the predictors of $\mathrm{AF}$ recurrence. The predictive model was established based on the results of the logistics regression and was visualized by the "Rms" package under the R language platform (version 4.0.3). The "nomogramFormula" package was used to calculate the total score of each patient. Calibration plot and receiver operating characteristic (ROC) curve were used separately to evaluate the calibration and discrimination of the predictive model. Youden's index was calculated by the formula $\mathrm{J}=$ sensitivity+specificity -1 . Kaplan-Meier plot was performed to compare the rate of freedom from AF recurrence after surgery. Spearman's rank correlation coefficient was used to reveal the relationship between circRNAs and clinical characteristics and was

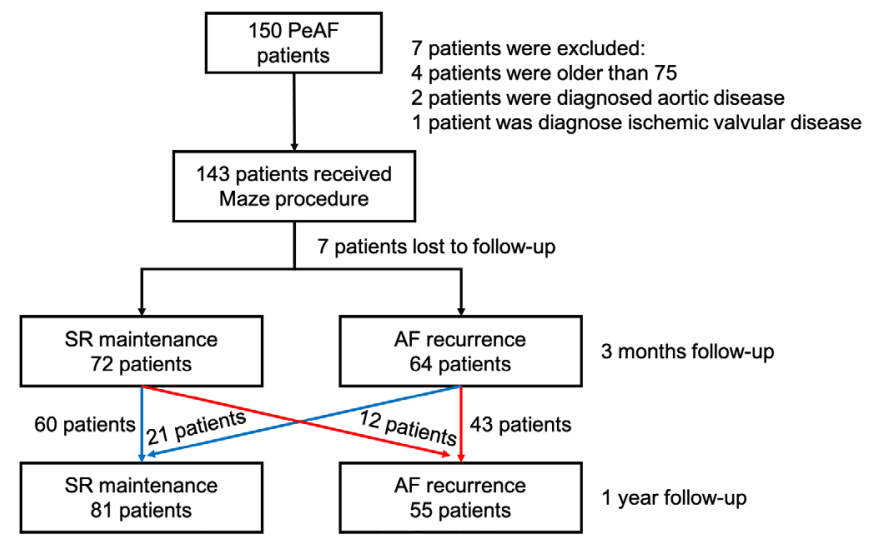

Figure 1. Flow chart diagram of patients. Total of 150 PeAF patients were enrolled, seven patients were excluded based on the exclusion criteria, another seven patients were excluded because of loss to follow up. The data of the remaining 136 patients were used for further analysis. The blue arrow represents patients restored sinus rhythm, and the red arrow represents patients suffering from $A F$ recurrence. $A F$, atrial fibrillation; PeAF, persistent atrial fibrillation; SR, sinus rhythm

visualized by "corrplot" package. $P$-value less than 0.05 was considered significant.

\section{RESULTS}

Patients' characteristics: A total of 150 persistent AF patients with VHD were admitted to our center from September 2018 to June 2019. Seven patients were excluded from this study, according to the exclusion criteria. Among them, four patients were over age 75, two patients were diagnosed with aortic disease, and another one patient was excluded because of ischemic valve disease. The remaining 143 patients were put forward to receive surgical ablation during operation, and all underwent the same ablation procedure. No perioperative mortality was reported. There were seven cases lost to follow up. Finally, data of 136 patients remained for further analysis and a flow chart diagram is shown in Figure 1. (Figure 1) The average follow-up time was $19.8 \pm 2.9$ months.

Patients were divided into two groups at three months or in one year after operation separately. There were 64 patients (47.1\%) suffering from recurrent AF (AFR1 group) in the first 3 -month follow-up period. LAD $(5.1 \pm 0.9 \mathrm{~cm}$ vs $5.5 \pm 1.1 \mathrm{~cm}$, $P=0.024)$ and $\mathrm{AF}$ duration $(41.0 \pm 60.6$ months vs $61.2 \pm 77.6$ months, $P=0.023$ ) were significantly different between the two groups. (Table 1)

According to the one-year results of the Holter examination, 81 patients restored sinus rhythm (SRM2 group), and the other 55 patients experienced recurrent atrial tachycardia (AFR2 group). Of the recurrent atrial tachycardia, 74.5\% were persistent AF. Compared with the follow-up results of three months, 21 patients in AFR1 group restored sinus rhythm, and 12 patients in SRM1 group progressed to persistent $\mathrm{AF}$ in one-year examination (Figure 1). Patients in SRM2 group were 4.6 years younger $(58.2 \pm 9.3$ vs $62.8 \pm 7.7, P$ 
Table 2. Clinical characteristics of valvular PeAF patients in one-year follow up

\begin{tabular}{|c|c|c|c|}
\hline Age (year) & $58.2 \pm 9.3$ & $62.8 \pm 7.7$ & 0.002 \\
\hline Body mass index (kg/m2) & $23.9 \pm 3.6$ & $23.7 \pm 3.4$ & 0.849 \\
\hline AF duration (month) & $35.2 \pm 54.8$ & $67.2 \pm 72.9$ & $<0.001$ \\
\hline Hypertension (n, \%) & $26(32.1 \%)$ & $21(38.2 \%)$ & 0.582 \\
\hline Diabetes mellitus (n, \%) & $6(7.4 \%)$ & $2(3.6 \%)$ & 0.473 \\
\hline Chronic kidney disease (n, \%) & $2(2.5 \%)$ & $1(1.8 \%)$ & 1.000 \\
\hline Cholesterol (n, \%) & $1.35 \pm 0.69$ & $1.63 \pm 3.57$ & 0.051 \\
\hline Triglyceride (n, \%) & $3.80 \pm 0.91$ & $3.65 \pm 0.78$ & 0.314 \\
\hline Stroke (n, \%) & $4(4.9 \%)$ & $3(5.5 \%)$ & 1.000 \\
\hline \multicolumn{4}{|l|}{ Transthoracic echocardiography } \\
\hline Left atria diameter $(\mathrm{cm})$ & $5.18 \pm 0.86$ & $5.39 \pm 1.12$ & 0.400 \\
\hline Ejection fraction (\%) & $53.1 \pm 6.7$ & $52.0 \pm 8.1$ & 0.385 \\
\hline Rheumatic valvular disease (n, \%) & $43(53.1 \%)$ & $29(52.7 \%)$ & 1.000 \\
\hline \multicolumn{4}{|l|}{ NYHA† Class } \\
\hline Class I & 3 & 2 & \\
\hline Class II & 38 & 20 & 0.511 \\
\hline
\end{tabular}

$=0.002)$ than the AFR2 group with shorter AF duration time (35.2 \pm 54.8 months vs $67.2 \pm 72.9$ months, $P<0.001)$. No difference was found in other variables. (Table 2)

The relationship between circRNAs and AF recurrence: The relative expression of circ 81906-RYR $(0.0160 \pm 0.008$ vs. $0.0245 \pm 0.0141, P<0.001)$, circ $44782-$ LAMA2 $(0.00564 \pm 0.00290$ vs. $0.00754 \pm 0.00384, P=0.002)$, circ $418-\mathrm{KCNN} 2 \quad(0.00760 \pm 0.00475$ vs. $0.01071 \pm 0.00812$, $P=0.011)$, and circ 35880 -ANO5 $(0.0242 \pm 0.0111$ vs. $0.0341 \pm 0.0178, P<0.001)$ were found significantly increased in the AFR2 group patients compared with the SRM2 group. (Figure 2) (Table 3)

Furthermore, ROC curve was used to evaluate the relationship between specific circRNA and AF recurrence. The results showed that circ 81906-RYR2 (AUC: 0.699, 95\% CI: 0.609-0.789, $P<0.001$ ), circ 35880-ANO5 (AUC: $0.673,95 \%$ CI: $0.580-0.766, P<0.001$ ), circ 44782-LAMA2 (AUC: 0.673, 95\% CI: $0.548-0.739, P<0.01$ ), and circ $417-\mathrm{KCNN} 2$ (AUC: 0.614, 95\% CI: $0.518-0.710, P<0.05$ ) could distinguish $\mathrm{AF}$ recurrence patients to SR restoration patients. (Figure 3)

Correlations of circRNAs with clinical characteristics: The Spearman's correlation rank coefficient revealed that circ $418-\mathrm{KCNN} 2(P=0.002)$ and $\operatorname{circ} 255-\mathrm{ITGA} 7(P=0.001)$ were negatively and circ 44782-LAMA2 $(P=0.042)$ was positively related with LAD. Circ $81906-\mathrm{RYR} 2(P=0.006)$ was positively correlated with $\mathrm{AF}$ duration. No correlation of circRNAs were found with age, EF, BNP, and CRP in PeAF patients. (Figure 4)

Risk factors of AF recurrence after surgical ablation: Data of the one-year follow up were used to evaluate the risk factors of AF recurrence. Univariate analysis demonstrated that advanced age $(P=0.004)$, longer $\mathrm{AF}$ duration $(P=0.007)$, increased expression of circ 81906-RYR2 $(P<0.001)$, circ 44782-LAMA2 $(P=0.003)$, circ $418-\mathrm{KCNN} 2(P=0.013)$, and circ 35880-ANO5 $(P=0.001)$ were risk factors for $\mathrm{AF}$ recurrence. (Table 4) Further multivariate analysis revealed that age (OR=1.071, 95\% CI: $1.019-1.125, P=0.007), \mathrm{AF}$ duration (OR=1.007, 95\% CI: $1.000-1.013, P=0.040)$, and circ 81906-RYR2 (OR=2.213, 95\% CI: 1.428-3.429, $P<$ 0.001 ) were significant independent predictors for AF recurrence (Table 4). The predictive model was established according to the results of the logistics regression analysis and was visualized by the nomogram. (Figure 5)

Calibration and discrimination analysis of the predictive model: The calibration plot and ROC curve was used to examine the calibration and discrimination of the predictive model separately. There was no difference between the predicted and observed value of the predictive model $(P=$ 0.328 , Figure 5B). The AUC of the predictive model was 0.77 (95\% CI: 0.690-0.849, $P<0.001$ ) (Figure 5C). The Youden's 


\begin{tabular}{|c|c|c|c|}
\hline Class III & 39 & 31 & \\
\hline Class IV & 1 & 2 & \\
\hline $\mathrm{BNP} \ddagger(\mathrm{pg} / \mathrm{mL})$ & $316.5 \pm 264.0$ & $357.2 \pm 347.8$ & 0.980 \\
\hline eGFRT $\left(\mathrm{mL} /\left(\mathrm{min}^{*} 1.73 \mathrm{~m}^{2}\right)\right.$ & $93.2 \pm 22.8$ & $96.1 \pm 22.5$ & 0.558 \\
\hline \multicolumn{4}{|l|}{ Intraoperative data } \\
\hline Aortic cross-clamp time (min) & $117.4 \pm 43.3$ & $109.2 \pm 40.3$ & 0.235 \\
\hline Mitral valve repair (n) & 23 & 17 & \\
\hline Mitral valve replacement (n) & 35 & 31 & 0.691 \\
\hline Aortic valve repair (n) & 7 & 3 & 1.000 \\
\hline Coronary artery bypass graft ( $n$ ) & 7 & 5 & 1.000 \\
\hline Bioprosthetic valve (n) & 37 & 27 & 0.107 \\
\hline Mechanical valve (n) & 23 & 7 & \\
\hline \multicolumn{4}{|l|}{ Types of arrhythmia } \\
\hline Persistent atrial fibrillation (n, \%) & $41(74.5 \%)$ & & \\
\hline Paroxysmal atrial fibrillation (n, \%) & $6(10.9 \%)$ & & \\
\hline Atrial flutter (n, \%) & $8(14.5 \%)$ & & \\
\hline Total score $>70$ points $(n, \%)$ & $26(32.1 \%)$ & $40(72.7 \%)$ & $<0.001$ \\
\hline
\end{tabular}

łBNP, brain natriuretic peptide; §CRP, C-reactive protein; $₫$ eGFR, estimated glomerular filtration rate; †NYHA, New York heart association

index of the total score in the predictive model was 70 points with sensitivity of $87.3 \%$ and specificity of $55.6 \%$ (Figure $5 \mathrm{C}$ red point). Clinical data of all patients was regrouped by the Youden's index. Kaplan-Meier analysis demonstrated that patients with total score more than 70 points showed high AF recurrence rate in one year after surgery $(50 \%$ vs. $20 \%, P<$ 0.001). (Figure 6)

\section{DISCUSSION}

Surgical ablation should be recommended in patients with symptomatic AF during valve surgery. LAA excision is suggested to reduce the incidence of AF induced thrombotic events [Baumgartner 2017]. Surgical technique improvements were achieved to decrease the postoperative AF recurrence, however, the outcome of surgical ablation still is not satisfactory in AF patients with VHD, especially those with rheumatic valvular diseases [Kong 2010]. A limited number of studies have reported that AF duration, left atrial size, and the severity of mitral regurgitation were independent risk factors for AF recurrence in valvular AF patients [Zhao 2014; Mulukutla 2018].

CircRNA is a kind of non-coding RNA and is derived from the process of alternative splicing of pre-mRNAs [Jeck
2014; Jeck 2013]. Researchers have demonstrated that circRNAs can act as a microRNA sponge to regulate miRNA function [Memczak 2013], bind with proteins to regulate the interaction between proteins [Ashwal-Fluss 2014], and form peptide to participate different biological process [Abe 2015]. Recently, circRNAs have proven to act as clinical biomarkers in central nervous diseases [Westholm 2014] and cancers [Han 2018], however, the function of circRNAs in AF still remains poorly understood. In our former study, we described the expression profile of circRNAs in AF patients with VHD and validated 10 differentially expressed circRNAs in patients' LAA tissue [Zhu 2020]. To our knowledge, this was the first study to evaluate the relationship between LAA circRNAs and $\mathrm{AF}$ recurrence after surgical ablation.

According to our previous study [Zhu 2020], the five most differentially expressed circRNAs were selected in this study. We found the recurrence rate of valvular persistent AF patients was $40.4 \%$ in our center. The recurrence rate was higher than other studies because of the larger preoperative left atrial diameter of patients enrolled in this study (the average left atrial diameter was $5.2 \mathrm{~cm}$ ) and the large amount of rheumatic valvular disease patients $(52.9 \%)$. We found that increased age $(P=0.002)$ and longer AF duration $(P<$ $0.001)$ were found in the AF recurrence group. The qPCR results showed that four circRNAs (circ 81906-RYR2, circ 
A

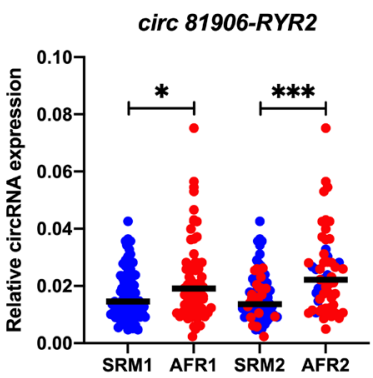

D

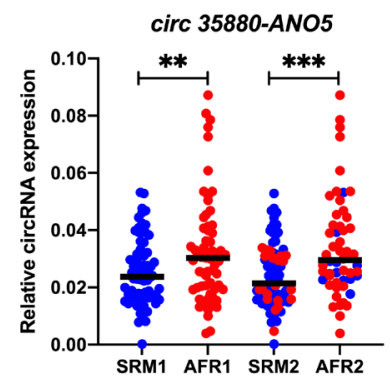

B

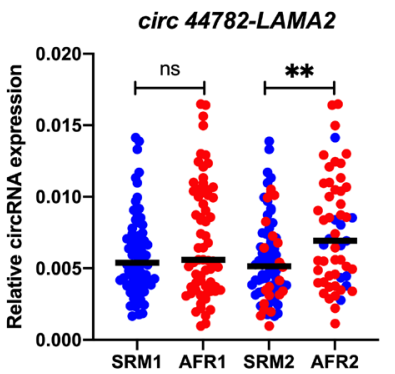

E

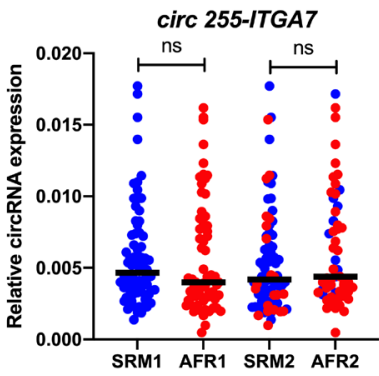

C

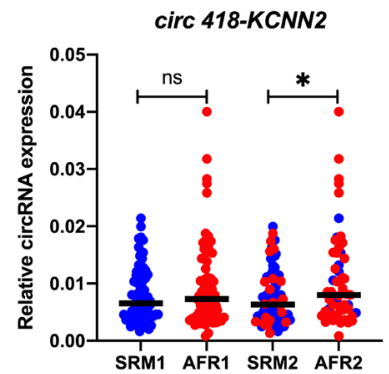

Figure 2. Relative expression of circRNAs in left atrial appendage in valvular PeAF patients. A) The relative expression of circ 81906-RYR2 was increased in both the AFR1 $(P=0.013)$ and AFR2 group $(P<0.001)$. B) The relative expression of circ 44782-LAMA2 was increased in the AFR2 group $(P=0.002)$. C) The relative expression of circ 418-KCNN2 was increased in the AFR2 group $(P=0.011)$. D) The relative expression of circ $35880-A N O 5$ was increased in both the AFR1 $(P=0.009)$ and AFR2 group $(P<0.001)$. E) The relative expression of circ $255-$ ITGA7 was not significantly different between both groups. Blue dots mean patients in the SRM1 group and red dots mean patients in the AFR1 group. ${ }^{*} P<0.05, * * P<0.01, * * * P<0.001$. AFR, atrial fibrillation recurrence; SRM, sinus rhythm maintenance

Table 3. Expression of circRNA in left atria appendage

\begin{tabular}{lcccccc}
\hline & SRM1 $(N=72)$ & AFR1 $(N=64)$ & $P$-value & SRM2 $(N=81)$ & AFR2 $(N=55)$ & $P$-value \\
\hline circ 81906-RYR2 & $0.0171 \pm 0.009$ & $0.0220 \pm 0.0140$ & 0.013 & $0.0160 \pm 0.008$ & $0.0245 \pm 0.0141$ \\
circ 44782-LAMA2 & $0.00595 \pm 0.00287$ & $0.00662 \pm 0.00379$ & 0.069 & $0.00564 \pm 0.00290$ & $0.00754 \pm 0.00384$ & 0.002 \\
circ 418-KCNN2 & $0.00976 \pm 0.0120$ & $0.00923 \pm 0.00635$ & 0.107 & $0.00760 \pm 0.00475$ & $0.01071 \pm 0.00812$ & 0.011 \\
circ 35880-ANO5 & $0.0266 \pm 0.0165$ & $0.0302 \pm 0.0174$ & 0.009 & $0.0242 \pm 0.0111$ & $0.0341 \pm 0.0178$ & $<0.001$ \\
circ 255-ITGA7 & $0.00582 \pm 0.00358$ & $0.00863 \pm 0.0133$ & 0.881 & $0.00542 \pm 0.00353$ & $0.00621 \pm 0.00399$ & 0.230
\end{tabular}

44782-LAMA2, circ 418-KCNN2, and circ 35880-ANO5) increased significantly in AF recurrence patients. These circRNAs could distinguish the AF recurrence patients with SR restoration patients by ROC curve analysis. However, the diagnostic efficacy was inadequate with circRNA alone, according to the AUC value of each circRNA (range from 0.6 to 0.7 ). Therefore, multivariate analysis was performed to conduct a predictive model for AF recurrence by using logistic regression. The results showed that increased expression of circ 81906-RYR2 was an independent risk factor for $\mathrm{AF}$ recurrence, besides advanced age and longer AF duration which have been reported in other studies [Zhao 2014; Iwamura 2011]. The results of the calibration plot and ROC curve showed this model was consistent well with the actual data and had good diagnostic efficacy. Nomogram was used to visualize the predictive model and the total score of each patient was calculated. The Kaplan-Meier plot based on the Youden's index of the predictive model also proved that patients with total score over 70 points were at high risk to experience AF recurrence during follow up. On the other hand, patients under 70 points could be considered at low risk of AF recurrence after surgery. For example, if a 50-year-old male patient with five years AF history was admitted and the expression of circ 81906-RYR2 was 0.01 , his total score would be 50.5 points and the AF recurrence rate in one year would be $18 \%$. In a word, he would be considered a low-risk patient.

Themistoclakis and Maroto et al. reported that recurrence of atrial tachycardias three months after ablation were associated with $\mathrm{AF}$ late recurrence after the blanking period [Themistoclakis 2008; Maroto 2011]. In this study, 64 patients (47.1\%) experienced atrial tachyarrhythmias in three months after surgery, and $43(67.2 \%)$ among them remain AF until one year after surgery. We also found that 21 patients in the 

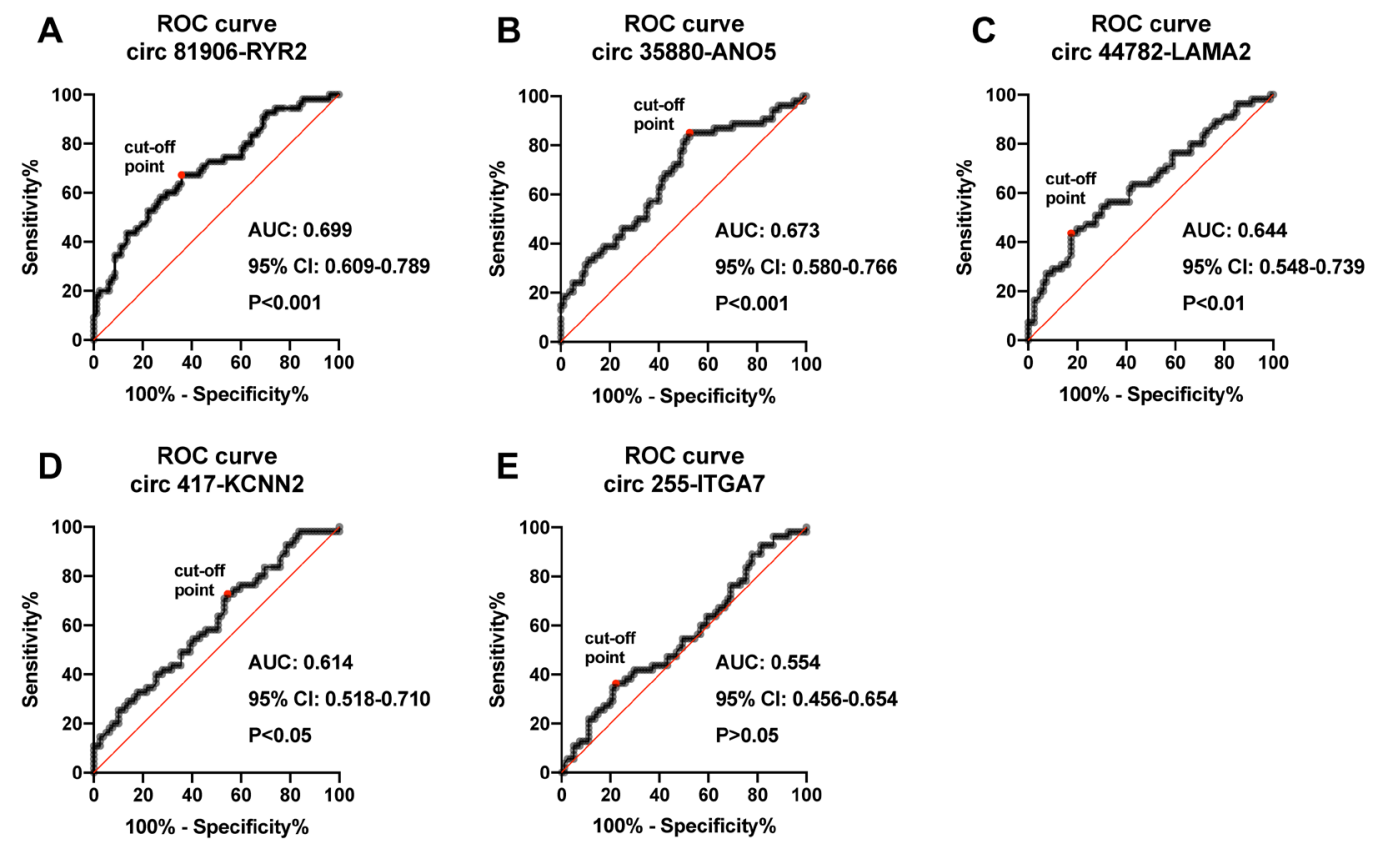

Figure 3. ROC curve of circRNAs for predicting AF recurrence after surgical ablation. ROC curve of circ 81906-RYR2, circ 35880-ANO5, circ 44782-LAMA2, circ 417-KCNN2, and circ 255-ITGA7. The red dot means cut-off point of each ROC curve.

AFR1 group restored sinus rhythm and 12 patients in the SRM1 group relapsed into AF one year after surgery. After calculating the predicted points of the AFR1 patients, there were 28 patients under 70 points, and $16(57.1 \%)$ of them restored sinus rhythm in one year. For those low-risk patients (total score less than 70 points) who experienced atrial tachycardias three months after surgery, early interventions, such as cardioversion and antiarrhythmic drugs therapy, were effective to help restore sinus rhythm in one year. It is worth mentioning that redefining the blanking period of surgical ablation is needed in the future as other research has reported [Alipour 2017; Xia 2020]. This kind of predictive model would be helpful with early intervention for patients in the blanking period.

CircRNA proved to have the potential as a clinical biomarker, according to the following properties. First, the circular structure ensures the biological stability of circRNA [Suzuki 2014]. The half-life of most circRNAs is longer than 48 hours, which helps them to be detected in different kinds of diseases [Jeck 2014]. Second, the expression of some circRNAs is tissue-specific. For instance, circRNAs from RMST and KLHL2 are highly expressed in the brain than other tissues [You 2015]. Some of them are enriched in synaptoneurosome compared with the whole-brain lysate [Rybak-Wolf 2015]. Our previous research also found the heart specific expression of circRNAs from RYR2, especially circ 81906-RYR2 [Zhu 2020]. Third, the expression of circRNA is related to the disease progression, such as cancer [Shang 2016; Yao 2017] or aging-related diseases [Yang 2018]. In this study, we found the expression of circ 81906RYR2 was positively related to the AF duration $(P=0.006)$. To summarize, the detectability, heart specific expression,

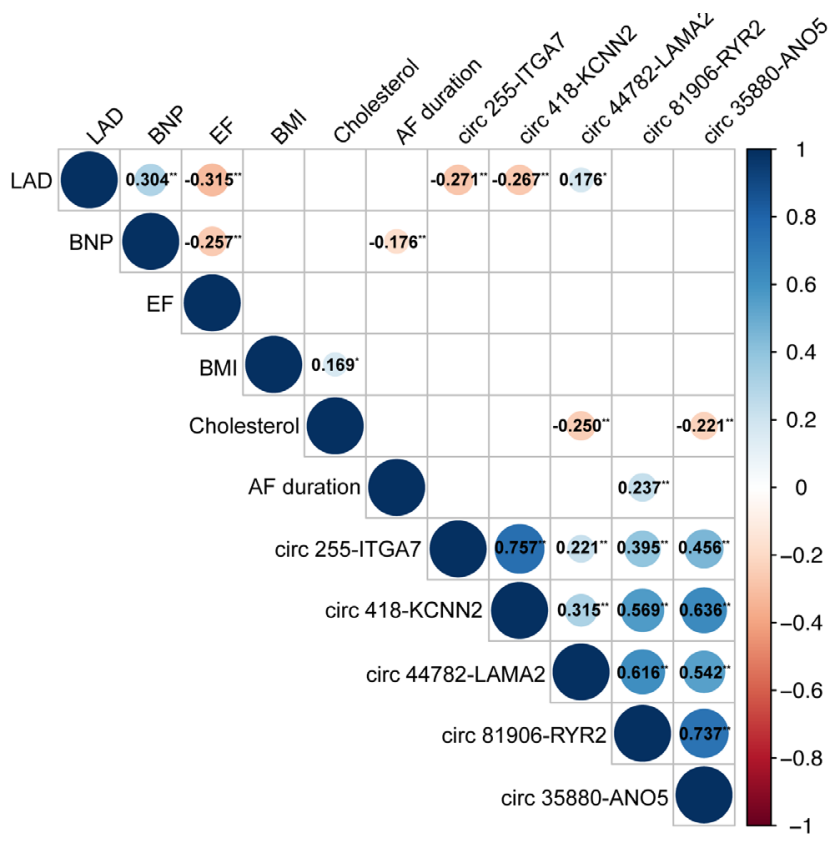

Figure 4. Correlations of circRNAs with clinical characteristics. Spearman's rank correlation coefficient was visualized by “corrplot” package. Blue circular means positive relevant and red circular means negative relevant. The correlation coefficient was listed in each intersection cell which relates to the corresponding row and column items. ${ }^{*} P<0.05$, $* * P<0.01$. AF, atrial fibrillation; BMl, body mass index; BNP, brain natriuretic peptide; EF, ejection fraction; LAD, left atrial diameter. 
Table 4. Univariate and multivariate analysis of risk factors for recurrence of AF after surgical ablation

\begin{tabular}{|c|c|c|c|c|c|c|}
\hline Variables & OR & $95 \% \mathrm{Cl}$ & $P$-value & OR & $95 \% \mathrm{Cl}$ & $P$-value \\
\hline $\mathrm{AF}$ duration & 1.008 & $1.002-1.014$ & 0.007 & 1.007 & $1.000-1.013$ & 0.036 \\
\hline circ 81906-RYR2 & 2.283 & $1.502-3.470$ & $<0.001$ & 2.210 & $1.425-3.427$ & $<0.001$ \\
\hline circ 418-KCNN2 & 1.603 & $1.103-2.328$ & 0.013 & & & \\
\hline circ 35880-ANO5 & 1.997 & $1.336-2.983$ & 0.001 & & & \\
\hline
\end{tabular}

A

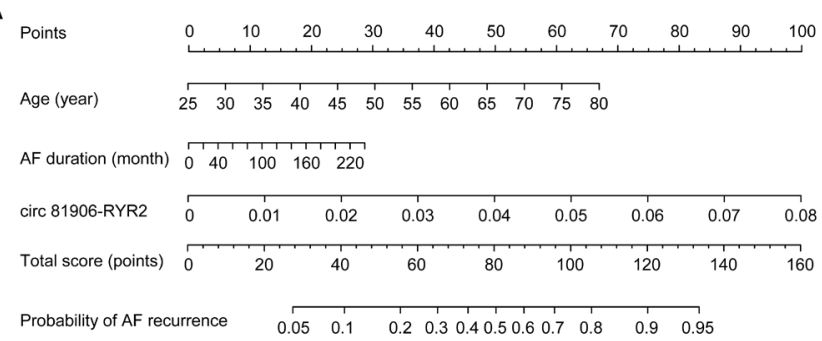

B

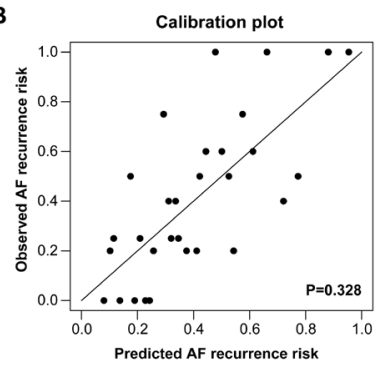

C

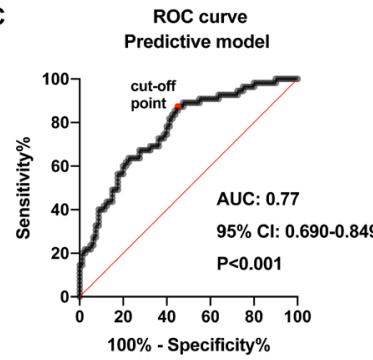

Figure 5. Visualization, calibration, and discrimination of the predictive model. A) Nomogram of the predictive model. Each value of risk factor could be converted into a detail point, and the total score would reflect the probability of atrial fibrillation recurrence in one-year follow up. B) Calibration plot of the predictive model. $X$-axis represented predicted value and $\mathrm{Y}$-axis represented observed value. C) ROC curve of the predictive model. The red dot means cut-off point of the predictive model. $\mathrm{AF}$, atrial fibrillation

and relationship with disease progression make it possible for circ 81906-RYR2 to become a new-found clinical biomarker for AF recurrence.

Limitations: Several limitations of this study should be mentioned. First, the patient number and the follow-up length of this study is limited. More AF patients with VHD and longer follow-up time are needed to make the validation of the predictive model. Second, LAA tissue is necessary for the detection of circ 81906-RYR2; this kind of detection is restricted to surgical ablation. For patients receiving catheter ablation, extra LAA section is needed to get the LAA tissue. Third, AF recurrence is always asymptomatic and undetectable; a long-term heart rhythm monitor is recommended.

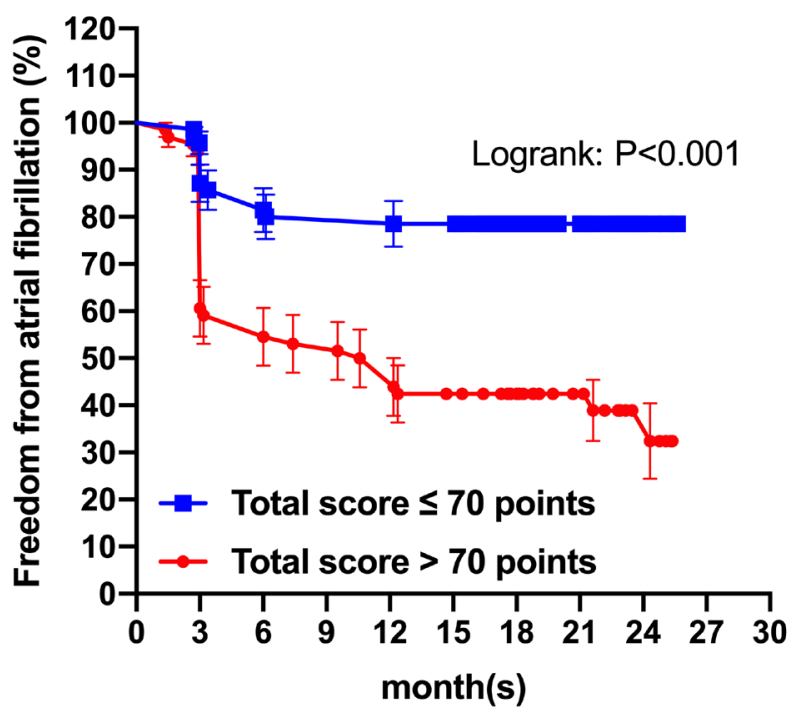

Figure 6. Kaplan-Meier plots of freedom from atrial fibrillation recurrence, according to the predictive model. Patients with a total score of less than 70 points showed a high rate of freedom from AF recurrence in one-year follow up ( $80 \%$ vs. $50 \%, \mathrm{P}<0.001)$.

\section{CONCLUSION}

Left atrial appendage circular RNA circ 81906-RYR2 was an independent risk factor for AF recurrence after surgical ablation. A predictive model that consists of age, AF duration, and circ 81906-RYR2 may be an alternative for prediction of $\mathrm{AF}$ recurrence. What's more, early intervention for low-risk patients is helpful to the restoration of sinus rhythm during follow up.

\section{ACKNOWLEDGEMENTS}

This work was supported by the National Natural Science Foundation of China [81970401 to Dongjin Wang, 81800241 to Ran Mo]; and the Jiangsu Provincial Medical Youth Talent [QNRC2016035 to Qing Zhou]. The authors thank Dr. 
Yuzhou Lu and Dr. Shi Xiong, of the Department of Cardiothoracic Surgery, Drum Tower Hospital Affiliated to Nanjing University Medical School, for surgical samples collection.

\section{REFERENCES}

Abe N, Matsumoto K, Nishihara M, et al. 2015. Rolling Circle Translation of Circular RNA in Living Human Cells. Sci Rep. 5:16435.

Alipour P, Azizi Z, Pirbaglou M, et al. 2017. Defining Blanking Period Post-Pulmonary Vein Antrum Isolation. JACC Clin Electrophysiol. 3:568-76.

Ashwal-Fluss R, Meyer M, Pamudurti NR, et al. 2014. circRNA biogenesis competes with pre-mRNA splicing. Mol Cell. 56:55-66.

Balk EM, Garlitski AC, Alsheikh-Ali AA, et al. 2010. Predictors of atrial fibrillation recurrence after radiofrequency catheter ablation: a systematic review. J Cardiovasc Electrophysiol. 21:1208-16.

Baumgartner H, Falk V, Bax JJ, et al. 2017. 2017 ESC/EACTS Guidelines for the management of valvular heart disease. Eur Heart J. 38:2739-91.

Chen X, Dong JZ, Du X, et al. 2018. Long-term outcome of catheter ablation for atrial fibrillation in patients with apical hypertrophic cardiomyopathy. J Cardiovasc Electrophysiol. 29:951-7.

Fayad G, Le Tourneau T, Modine T, et al. 2005. Endocardial radiofrequency ablation during mitral valve surgery: effect on cardiac rhythm, atrial size, and function. Ann Thorac Surg. 79:1505-11.

Gammie JS, Haddad M, Milford-Beland S, et al. 2008. Atrial fibrillation correction surgery: lessons from the Society of Thoracic Surgeons National Cardiac Database. Ann Thorac Surg. 85:909-14.

Geidel S, Ostermeyer J, Lass M, et al. 2006. Permanent atrial fibrillation ablation surgery in CABG and aortic valve patients is at least as effective as in mitral valve disease. Thorac Cardiovasc Surg. 54:91-5.

Gillinov AM, Bakaeen F, McCarthy PM, et al. 2006. Surgery for paroxysmal atrial fibrillation in the setting of mitral valve disease: a role for pulmonary vein isolation? Ann Thorac Surg. 81:19-26; discussion 7-8.

Han B, Chao J, Yao H. 2018. Circular RNA and its mechanisms in disease: From the bench to the clinic. Pharmacol Ther. 187:31-44.

Iwamura T, Kajimoto K, Yamamoto T, et al. 2011. Mid-term results for the Maze procedure in patients with non-mitral valvular atrial fibrillation. Ann Thorac Cardiovasc Surg. 17:356-62.

Jeck WR, Sharpless NE. 2014. Detecting and characterizing circular RNAs. Nat Biotechnol. 32:453-61.

Jeck WR, Sorrentino JA, Wang K, et al. 2013. Circular RNAs are abundant, conserved, and associated with ALU repeats. RNA. 19:141-57.

Khan MS, Yamashita K, Sharma V, et al. 2020. Perioperative Biomarkers Predicting Postoperative Atrial Fibrillation Risk After Coronary Artery Bypass Grafting: A Narrative Review. J Cardiothorac Vasc Anesth. 34:1933-41.

Kong MH, Lopes RD, Piccini JP, et al. 2010. Surgical Maze procedure as a treatment for atrial fibrillation: a meta-analysis of randomized controlled trials. Cardiovasc Ther. 28:311-26.

Maroto LC, Carnero M, Silva JA, et al. 2011. Early recurrence is a predictor of late failure in surgical ablation of atrial fibrillation. Interact Cardiovasc Thorac Surg. 12:681-6.

Memczak S, Jens M, Elefsinioti A, et al. 2013. Circular RNAs are a large class of animal RNAs with regulatory potency. Nature. 495:333-8.

Mulukutla S, Althouse AD, Jain SK, Saba S. 2018. Increased left atrial size is associated with higher atrial fibrillation recurrence in patients treated with antiarrhythmic medications. Clin Cardiol. 41:825-9.

Nigro JM, Cho KR, Fearon ER, et al. 1991. Scrambled exons. Cell. 64:607-13.

Pallisgaard JL, Gislason GH, Hansen J, et al. 2018. Temporal trends in atrial fibrillation recurrence rates after ablation between 2005 and 2014: a nationwide Danish cohort study. Eur Heart J. 39:442-9.

Rattka M, Pott A, Kuhberger A, et al. 2020. Restoration of sinus rhythm by pulmonary vein isolation improves heart failure with preserved ejection fraction in atrial fibrillation patients. Europace. 22:1328-36.

Robertson JO, Saint LL, Leidenfrost JE, Damiano RJ, Jr. 2014. Illustrated techniques for performing the Cox-Maze IV procedure through a right mini-thoracotomy. Ann Cardiothorac Surg. 3:105-16.

Rybak-Wolf A, Stottmeister C, Glazar P, et al. 2015. Circular RNAs in the Mammalian Brain Are Highly Abundant, Conserved, and Dynamically Expressed. Mol Cell. 58:870-85.

Shang X, Li G, Liu H, et al. 2016. Comprehensive Circular RNA Profiling Reveals That hsa_circ_0005075, a New Circular RNA Biomarker, Is Involved in Hepatocellular Crcinoma Development. Medicine (Baltimore). $95: \mathrm{e} 3811$.

Suzuki H, Tsukahara T. 2014. A view of pre-mRNA splicing from RNase R resistant RNAs. Int J Mol Sci. 15:9331-42.

Themistoclakis S, Schweikert RA, Saliba WI, et al. 2008. Clinical predictors and relationship between early and late atrial tachyarrhythmias after pulmonary vein antrum isolation. Heart Rhythm. 5:679-85.

Westholm JO, Miura P, Olson S, et al. 2014. Genome-wide analysis of drosophila circular RNAs reveals their structural and sequence properties and age-dependent neural accumulation. Cell Rep. 9:1966-80.

Xia Y, Liu J, Jia Y, et al. 2020. Redefining the Blanking Period by a LongTerm Follow-Up after Atrial Fibrillation Ablation Using Second-Generation Cryoballoon. Int Heart J. 61:936-43.

Yang D, Yang K, Yang M. 2018. Circular RNA in Aging and Age-Related Diseases. Adv Exp Med Biol. 1086:17-35.

Yao JT, Zhao SH, Liu QP, et al. 2017. Over-expression of CircRNA_100876 in non-small cell lung cancer and its prognostic value. Pathol Res Pract. 213:453-6.

You X, Vlatkovic I, Babic A, et al. 2015. Neural circular RNAs are derived from synaptic genes and regulated by development and plasticity. Nat Neurosci. 18:603-10.

Zhao L, Jiang W, Zhou L, et al. 2014. The role of valvular regurgitation in catheter ablation outcomes of patients with long-standing persistent atrial fibrillation. Europace. 16:848-54.

Zhu X, Tang X, Chong H, et al. 2020. Expression Profiles of Circular RNA in Human Atrial Fibrillation With Valvular Heart Diseases. Front Cardiovasc Med. 7:597932. 